\title{
Fixed point theorems for a class of generalized nonexpansive mappings
}

\section{Fatemeh Lael ${ }^{1 *}$ and Zohre Heidarpour ${ }^{2}$}

\section{"Correspondence:}

fatemehlael@yahoo.com

1 Department of Mathematics, Buein

Zahra Technical University, Buein

Zahra, Qazvin, Iran

Full list of author information is

available at the end of the article

\begin{abstract}
In this paper, we introduce a new class of generalized nonexpansive mappings. Some new fixed point theorems for these mappings are obtained.
\end{abstract}

MSC: $47 \mathrm{H} 10$

Keywords: monotone mapping; nonexpansive mapping; fixed point; $L_{p}$

\section{Introduction and preliminaries}

A nonexpansive mapping has a Lipschitz constant equal to 1 . The fixed point theory for such mappings is very rich [1-5] and has many applications in nonlinear functional analysis [6].

We first commence some basic concepts about generalization of nonexpansive mappings as formulated by Suzuki et al. [7, 8].

Definition 1 [8] Let $C$ be a nonempty subset of a Banach space $X$. We say that a mapping $T: C \rightarrow C$ satisfies condition $(C)$ on $C$ if $\frac{1}{2}\|x-T(x)\| \leq\|x-y\|$ implies $\|T(x)-T(y)\| \leq$ $\|x-y\|$, for $x, y \in C$.

Of course, every nonexpansive mapping satisfies condition $(C)$ but the converse is not correct and you can find some counterexamples for it in [8]. So the class of mappings which has condition $(C)$ is broader than the class of nonexpansive mappings.

In [7], condition $(C)$ is generalized as follows.

Definition 2 [7] Let $C$ be a nonempty subset of a Banach space $X$ and $\lambda \in(0,1)$. We say that a mapping $T: C \rightarrow X$ satisfies $\left(C_{\lambda}\right)$-condition on $C$ if $\lambda\|x-T(x)\| \leq\|x-y\|$ implies $\|T(x)-T(y)\| \leq\|x-y\|$, for $x, y \in C$.

So if $\lambda=\frac{1}{2}$, we will have condition $(C)$. There are examples that show the converse is false; see [7].

In [9], monotone nonexpansive mappings are defined in $L_{1}[0,1]$.

We next review some notions in $L_{p}[0,1]$. All of them can be found in [10].

Consider the Riesz Banach space $L_{p}[0,1]$, where $\int_{0}^{1}|f(x)|^{p} d x<+\infty$ and $p \in(0,+\infty)$. Also, we have $f=0$ when the set

$$
\{x \in[0,1]: f(x)=0\},
$$

(c) 2016 Lael and Heidarpour. This article is distributed under the terms of the Creative Commons Attribution 4.0 Internationa License (http://creativecommons.org/licenses/by/4.0/), which permits unrestricted use, distribution, and reproduction in any medium, provided you give appropriate credit to the original author(s) and the source, provide a link to the Creative Commons license, and indicate if changes were made. 
has Lebesgue measure zero. In this case, we say $f=0$ almost everywhere. An element of $L_{p}[0,1]$ is therefore seen as a class of functions. The norm of any $f \in L_{p}[0,1]$ is given by $\|f\|_{p}=\left(\int_{0}^{1}|f(x)|^{p} d x\right)^{\frac{1}{p}}$. Throughout this paper, we will write $L_{p}$ instead of $L_{p}[a, b], a, b \in \mathbb{R}$ and $\|\cdot\|$ instead of $\|\cdot\|_{p}$.

In this paper, we redefine Definition 2 on a subset of Banach space $L_{p}$ and those theorems which are proved in [9] generalize to a wider class of monotone $\left(C_{\lambda}\right)$-condition with preserving their fixed point property.

\section{Main results}

Let $C$ be a nonempty subset of $L_{p}$ which is equipped with a vector order relation $\preceq$. A map $T: C \rightarrow C$ is called monotone if for all $f \preceq g$ we have $T(f) \preceq T(g)$.

We generalize the $\left(C_{\lambda}\right)$-condition as follows.

Definition 3 Let $C$ be a nonempty subset of a Banach space $L_{p}$. For $\lambda \in(0,1)$, we say that a mapping $T$ monotone $\left(C_{\lambda}\right)$-condition on $C$ if $T$ is monotone and for all $f \preceq g, \lambda \| f-$ $T(f)\|\leq\| g-f \|$ implies $\|T(g)-T(f)\| \leq\|g-f\|$.

Note Definition 3 is a generalization of the monotone nonexpansive mapping which is defined in [9] as follows.

A map $T$ is said to be monotone nonexpansive if $T$ is monotone and for $f \preceq g$, we have $\|T(g)-T(f)\| \leq\|g-f\|$.

The next example is a direct generalization of monotone nonexpansive mapping.

Example 1 Let $C=\left\{f \in L_{p}[0,3]: f(x)=a\right\}$, where $a \in[0,3]$. For $f, g \in C$, consider the partial order relation

$$
f \preceq g \quad \text { iff } f(x) \leq g(x)
$$

Let $T: C \rightarrow C$ be defined by

$$
T(f)= \begin{cases}1, & f=3 \\ 0, & f \neq 3\end{cases}
$$

Then the mapping $T$ satisfies the monotone $\left(C_{\frac{1}{2}}\right)$-condition but it fails monotone nonexpansiveness. Indeed, whenever $f \preceq g$, if $0 \leq f(x) \leq g(x)<3$, then $\|T(f)-T(g)\| \leq\|f-g\|$. On the other hand, $0 \leq f(x)<3$ and $g=3$, so if $0 \leq f(x) \leq 2$ and $g=3$, then we have again $\|T(f)-T(g)\| \leq\|f-g\|$, but if $2<f(x)<3$ and $g=3$, then $\frac{1}{2}\|f\| \not \leq\|f-3\|$. Thus, the mapping $T$ satisfying monotone $\left(C_{\frac{1}{2}}\right)$-condition on $[0,3]$.

Let $f=2.9$ and $g=3$. Then $f \preceq g$ while $\|T(f)-T(g)\| \not \leq\|f-g\|$. Thus, $T$ is not monotone nonexpansive.

The following lemmas will be crucial to prove the main result of this paper.

Lemma 1 Let $C$ be convex and $T$ monotone. Assume that for some $f_{1} \in C, f_{1} \preceq T\left(f_{1}\right)$. Then the sequence $f_{n}$ defined by

$(\star) \quad f_{n+1}=\lambda T\left(f_{n}\right)+(1-\lambda) f_{n}$, 
$\lambda \in(0,1)$, satisfies

$$
f_{n} \preceq f_{n+1} \preceq T\left(f_{n}\right) \preceq T\left(f_{n+1}\right) .
$$

for $n \geq 1$.

Proof First, we prove that $f_{n} \preceq T\left(f_{n}\right)$. By assumption, we have $f_{1} \preceq T\left(f_{1}\right)$. Assume that $f_{n} \preceq$ $T\left(f_{n}\right)$, for $n \geq 1$. Then we have

$$
f_{n}=\lambda f_{n}+(1-\lambda) f_{n} \preceq \lambda T\left(f_{n}\right)+(1-\lambda) f_{n}=f_{n+1}
$$

i.e. $f_{n} \preceq f_{n+1}$. Since $T$ is monotone, $T\left(f_{n}\right) \preceq T\left(f_{n+1}\right)$. We have

$$
f_{n+1}=\lambda T\left(f_{n}\right)+(1-\lambda) f_{n} \preceq \lambda T\left(f_{n}\right)+(1-\lambda) T\left(f_{n}\right)=T\left(f_{n}\right) .
$$

Thus

$$
f_{n} \preceq f_{n+1} \preceq T\left(f_{n}\right) \preceq T\left(f_{n+1}\right),
$$

for $n \geq 1$. The proof is closely modeled on Lemma 3.1 of [9].

Note that under the assumption of Lemma 1, if we assume $T\left(f_{1}\right) \preceq f_{1}$, then we have

$$
T\left(f_{n+1}\right) \preceq T\left(f_{n}\right) \preceq f_{n+1} \preceq f_{n}
$$

for any $n \geq 1$.

A sequence $\left\{f_{n}\right\}$ in $C$ is called an almost fixed point sequence for $T$, if $\left\|f_{n}-T\left(f_{n}\right)\right\| \rightarrow 0$ (a.f.p.s. in short).

Lemma 2 Let $T: C \rightarrow L_{p}$ be a monotone $\left(C_{\lambda}\right)$-condition mapping and $f_{n}$ be a bounded a.f.p.s. for $T$. Then

$$
\liminf _{n}\left\|f_{n}-T(f)\right\| \leq \liminf _{n_{k}}\left\|f_{n}-f\right\|
$$

for $f \in C$ which $f_{n} \preceq f$ and $\liminf _{n}\left\|f_{n}-f\right\|>0$, for all $n \geq 1$.

Proof Fix $f \in C$ such that $f_{n} \preceq f$. Since $f_{n}$ is an a.f.p.s., for $\epsilon=\frac{1}{2} \liminf { }_{n}\left\|f_{n}-f\right\|$, there is $n_{0}$ such that $\left\|f_{n}-T\left(f_{n}\right)\right\|<\epsilon$, for all $n \geq n_{0}$. This implies that

$$
\lambda\left\|f_{n}-T\left(f_{n}\right)\right\| \leq\left\|f_{n}-T\left(f_{n}\right)\right\|<\epsilon<\left\|f_{n}-f\right\|,
$$

for all $n \geq n_{0}$. Since $T$ satisfies the monotone $\left(C_{\lambda}\right)$-condition, we have

$$
\left\|T\left(f_{n}\right)-T(f)\right\| \leq\left\|f_{n}-f\right\|
$$

for all $n \geq n_{0}$. So by the triangle inequality and (1), we have

$$
\left\|f_{n}-T(f)\right\| \leq\left\|f_{n}-T\left(f_{n}\right)\right\|+\left\|T\left(f_{n}\right)-T(f)\right\| \leq\left\|f_{n}-T\left(f_{n}\right)\right\|+\left\|f_{n}-f\right\| .
$$


Thus $\liminf _{n}\left\|f_{n}-T(f)\right\| \leq \liminf _{n}\left\|f_{n}-f\right\|$. The proof is closely modeled on Lemma 1 of [7].

Lemma 3 [11] If $\left\{f_{n}\right\}$ is a sequence of $L_{p}$-uniformly bounded functions on a measure space, and $f_{n} \rightarrow f$ almost everywhere, then

$$
\liminf _{n}\left\|f_{n}\right\|^{p}=\liminf _{n}\left\|f_{n}-f\right\|^{p}+\|f\|^{p}
$$

for all $p \in(0, \infty)$.

In the following, let $C$ be a nonempty, convex, and bounded set and $T: C \rightarrow C$ be a monotone $\left(C_{\lambda}\right)$-condition, for some $\lambda \in(0,1)$.

Theorem 1 Let $f_{1} \in C$ such that $f_{1} \preceq T\left(f_{1}\right)$. Then $f_{n}$ defined in $(\star)$ is an a.f.p.s.

Proof Since $f_{n+1}=\lambda T\left(f_{n}\right)+(1-\lambda) f_{n}$, for $n \geq 1$, we have

$$
\lambda\left\|f_{n}-T\left(f_{n}\right)\right\|=\left\|f_{n}-f_{n+1}\right\| .
$$

By Lemma 1, we have $f_{n} \preceq f_{n+1}$. Therefore, monotone $\left(C_{\lambda}\right)$-condition implies that $\| T\left(f_{n}\right)$ $T\left(f_{n+1}\right)\|\leq\| f_{n}-f_{n+1} \|$. Now, we can apply Lemma 3 of [1] to conclude that $\lim _{n}\left\|f_{n}-T\left(f_{n}\right)\right\|=$ 0 .

Example 2 We show that $T$, which is defined in Example 1, has an a.f.p.s. It is easy to see that $C$ is a nonempty, convex, and bounded subset of $L_{p}$. Also, we proved $T$ obeys the monotone $\left(C_{\frac{1}{2}}\right)$-condition. Moreover, $0 \preceq T(0)$. Thus, by Theorem 1, $T$ has an a.f.p.s.

Now, we construct an a.f.p.s. according $(\star)$. Let $f_{1}=0$. So $f_{n}=0$. Therefore

$$
\left\|f_{n}-T\left(f_{n}\right)\right\|=0 .
$$

Thus $f_{n}$ is an a.f.p.s.

Theorem 2 Let $C$ be compact. Assume there exists $f_{1} \in C$ such that $f_{1}$ and $T\left(f_{1}\right)$ are comparable. Then $T$ has a fixed point.

Proof Let $f_{n}$ be a sequence which is defined in $(\star)$. By Theorem $1, f_{n}$ is an a.f.p.s. Since $C$ is compact, $f_{n}$ has a convergent subsequence $f_{n_{k}}$ to $f$. By triangle inequality, we get

$$
\liminf _{n_{k}}\left\|T\left(f_{n_{k}}\right)-T(f)\right\| \leq \lim _{n_{k}}\left\|T\left(f_{n_{k}}\right)-f_{n_{k}}\right\|+\liminf _{n_{k}}\left\|f_{n_{k}}-T(f)\right\| .
$$

Since $f_{n}$ is an a.f.p.s., we have

$$
\liminf _{n_{k}}\left\|T\left(f_{n_{k}}\right)-T(f)\right\| \leq \liminf _{n_{k}}\left\|f_{n_{k}}-T(f)\right\| .
$$

Again, by triangle inequality, we have

$$
\liminf _{n_{k}}\left\|f_{n_{k}}-T(f)\right\| \leq \lim _{n_{k}}\left\|f_{n_{k}}-T\left(f_{n_{k}}\right)\right\|+\liminf _{n_{k}}\left\|T(f)-T\left(f_{n_{k}}\right)\right\| .
$$


Therefore,

$$
\liminf _{n_{k}}\left\|f_{n_{k}}-T(f)\right\| \leq \liminf _{n_{k}}\left\|T\left(f_{n_{k}}\right)-T(f)\right\| .
$$

From equations (2) and (3), we have

$$
\liminf _{n_{k}}\left\|f_{n_{k}}-T(f)\right\|=\liminf _{n_{k}}\left\|T\left(f_{n_{k}}\right)-T(f)\right\| .
$$

By using the partially order and convergent properties $f_{n_{k}} \preceq f$. Lemma 1 implies $f_{n_{k}} \preceq$ $f_{n_{k}+1} \preceq f$. So $\left\|f_{n_{k}+1}-f_{n_{k}}\right\| \leq\left\|f-f_{n_{k}}\right\|$. Since $f_{n_{k}+1}-f_{n_{k}}=\lambda\left(f_{n_{k}}-T\left(f_{n_{k}}\right)\right)$, we get

$$
\lambda\left\|f_{n_{k}}-T\left(f_{n_{k}}\right)\right\|=\left\|f_{n_{k}+1}-f_{n_{k}}\right\| .
$$

Therefore

$$
\lambda\left\|\left(f_{n_{k}}-T\left(f_{n_{k}}\right)\right)\right\| \leq\left\|f-f_{n_{k}}\right\| .
$$

Thus the monotone $\left(C_{\lambda}\right)$-condition implies

$$
\left\|T\left(f_{n_{k}}\right)-T(f)\right\| \leq\left\|f_{n_{k}}-f\right\| .
$$

Since $f_{n_{k}}$ is bounded, Lemma 3 implies

$$
\liminf _{n_{k}}\left\|f_{n_{k}}-T(f)\right\|=\liminf _{n_{k}}\left\|f_{n_{k}}-f\right\|+\|f-T(f)\| .
$$

From equation (4), we get

$$
\liminf _{n_{k}}\left\|f_{n_{k}}-f\right\|+\|f-T(f)\|=\liminf _{n_{k}}\left\|T\left(f_{n_{k}}\right)-T(f)\right\| .
$$

From equation (5), we get

$$
\underset{n_{k}}{\liminf }\left\|f_{n_{k}}-f\right\|+\|f-T(f)\| \leq \liminf _{n_{k}}\left\|f_{n_{k}}-f\right\|
$$

This implies that $T(f)=f$.

By Theorem 2, we can see that $T$ in Example 1, has a fixed point.

The following example shows that monotone $\left(C_{\lambda}\right)$-condition is a direct generalization of $\left(C_{\lambda}\right)$-condition.

Example 3 Let $C=\operatorname{co}\{x, \sin (x)\}$, where $x \in\left[-\frac{\pi}{2}, \frac{\pi}{2}\right]$. Define a partial order on $C$ as follows:

$$
f \preceq g \quad \text { iff } f(x) \leq g(x)
$$

Let $T: C \rightarrow C$ be

$$
T(f)= \begin{cases}\sin (x) & f \neq x \\ x & f=x\end{cases}
$$


Since $C$ is convex hull of a compact set $\{x, \sin (x)\}$, so it is a nonempty, convex and compact subset of $L_{p}$. Put $f=x$. Then $f$ and $T(f)$ are comparable. Also, $T$ obeys the monotone $\left(C_{\lambda}\right)$-condition. Thus, by Theorem $2, T$ has a fixed point.

Note, for $\lambda \in(0,1), T$ does not obey the $\left(C_{\lambda}\right)$-condition. Because, for $f=x$ and $g=\frac{x}{2}+$ $\frac{1}{2} \sin (x)$, we have $\lambda\|f-T(f)\| \leq\|f-g\|$, but $\|T(g)-T(f)\| \not \leq\|f-g\|$.

Theorem 3 Let $C$ be a weakly compact subset of $L_{2}$. Assume, there is $f_{1} \in C$ such that $f_{1} \preceq T\left(f_{1}\right)$. Then $T$ has a fixed point.

Proof By Theorem 1, $T$ has an a.f.p.s. $f_{n}$. Since $C$ is weakly compact, there is a weakly convergent subsequence $f_{n_{k}}$ to some $f \in C$. If $\liminf _{n_{k}}\left\|f_{n_{k}}-f\right\|=0$, then $f_{n_{k}}$ is convergent and we will have the same proof of Theorem 2. On the other hand, if $\liminf _{n_{k}}\left\|f_{n_{k}}-f\right\|>0$, then by Lemma 2 ,

$$
\liminf _{n_{k}}\left\|f_{n_{k}}-T(f)\right\| \leq \liminf _{n_{k}}\left\|f_{n_{k}}-f\right\| .
$$

We claim that $f=T(f)$. Because if $f \neq T(f)$, since $L_{2}$ satisfies Opial condition, we have

$$
\liminf _{n_{k}}\left\|f_{n_{k}}-f\right\|<\liminf _{n_{k}}\left\|f_{n_{k}}-T(f)\right\|
$$

which is a contradiction with inequality (6).

This result is a generalization of the original existence theorem in [7, 9] form monotone nonexpansive to monotone $\left(C_{\lambda}\right)$-condition. Therefore this class is bigger and is used to answer the question asked by $T$ Benavides [12]: Does $X$ also satisfy the fixed point property for Suzuki-type mappings?

\section{Competing interests}

The authors declare that they have no competing interests.

Authors' contributions

All authors contributed equally to the writing of this paper. All authors read and approved the final manuscript.

\section{Author details}

${ }^{1}$ Department of Mathematics, Buein Zahra Technical University, Buein Zahra, Qazvin, Iran. ${ }^{2}$ Department of Mathematics, Payame Noor University, Tehran, 19395-3697, Iran.

\section{Acknowledgements \\ The first author acknowledges Buein Zahra Technical University for supporting this research.}

Received: 13 February 2016 Accepted: 20 July 2016 Published online: 01 August 2016

\section{References}

1. Goebel, K, Kirk, WA: Iteration processes for nonexpansive mappings. Contemp. Math. 21, 115-123 (1983)

2. Ishikawa, S: Fixed points and iteration of a nonexpansive mapping in a Banach space. Proc. Am. Math. Soc. 59, 65-71 (1976)

3. Khan, AR, Hussain, N: Iterative approximation of fixed points of nonexpansive maps. Sci. Math. Jpn. 54, 503-511 (2001)

4. Kirk, WA: Fixed point theory for nonexpansive mappings. In: Fixed Point Theory. Lecture Notes in Mathematics, vol. 886, pp. 485-505 (1981)

5. Opial, Z: Weak convergence of the sequence of successive approximations for nonexpansive mappings. Bull. Am. Math. Soc. 73, 595-597 (1967)

6. Browder, FE: Nonexpansive nonlinear operators in a Banach space. Proc. Natl. Acad. Sci. USA 54, 1041-1044 (1965)

7. Falset, JG, Fuster, EL, Suzuki, T: Fixed point theory for a class of generalized nonexpansive mappings. J. Math. Anal. Appl. 375, 185-195 (2011)

8. Suzuki, T: Fixed point theorems and convergence theorems for some generalized nonexpansive mappings. J. Math Anal. Appl. 340, 1088-1095 (2008) 
9. Khamsi, MA, Khan, AR: On monotone nonexpansive mappings in $L_{1}([0,1])$. Fixed Point Theory Appl. 2015, Article ID 94 (2015). doi:10.1186/s13663-015-0346-x

10. Beauzamy, B: Introduction to Banach Spaces and Their Geometry. North-Holland, Amsterdam (1985)

11. Brezis, H, Lieb, E: A relation between pointwise convergence of functions and convergence of functionals. Proc. Am. Math. Soc. 88, 486-490 (1983)

12. Benavides, TD, Medina Perez, B: The fixed point property for some generalized nonexpansive mappings and renormings. J. Math. Anal. Appl. 429, 800-813 (2015)

Submit your manuscript to a SpringerOpen ${ }^{\circ}$ journal and benefit from:

- Convenient online submission

Rigorous peer review

- Immediate publication on acceptance

- Open access: articles freely available online

- High visibility within the field

- Retaining the copyright to your article 